Supporting Information:

\title{
SERS Amplification from Self-Organised Arrays of Plasmonic Nanocrescents
}

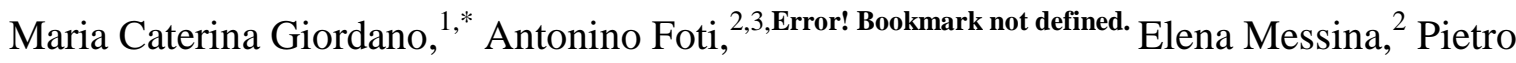 \\ Giuseppe Gucciardi, ${ }^{2}$ \\ Davide Comoretto, ${ }^{4}$ Francesco Buatier de Mongeot ${ }^{1, \#}$ \\ ${ }^{1}$ Dipartimento di Fisica, Università di Genova, Via Dodecaneso 33, I-16146 Genova, Italy \\ ${ }^{2}$ CNR IPCF Istituto per i Processi Chimico-Fisici, Viale F. Stagno D’Alcontres 37, I-98156 Messina, Italy \\ ${ }^{3}$ Dottorato di Ricerca in Fisica, Università di Messina, Viale F. Stagno D’Alcontres 31, I-98156 Messina, Italy \\ ${ }^{4}$ Dipartimento di Chimica e Chimica Industriale, Università di Genova Via Dodecaneso 31, 16146 , Genova , Italy
}

\section{\# Corresponding Author}

E-mail: buatier@fisica.unige.it

\section{* Author contributions}

M.C.G and A.F contributed equally to the manuscript. 
In Figure S1 (a),(b) we compare the optical spectra of Au nanocrescent arrays grown in different conditions; Au evaporation was respectively performed at $\theta=70^{\circ}$ (graph adapted from Ref. [Error! Bookmark not defined.]) and at $\theta=80^{\circ}$ (graph adapted from Error! Reference source not found.(d) of this paper). In Figure S1(a) we observe a High Energy mode (blue arrows), peaked around $650 \mathrm{~nm}$, which is excited for both TM and TE polarisation. On the other hand in Figure S1(b), due to the different evaporation conditions employed (i.e. $\theta=80^{\circ}$ ) the HE mode partially overlaps with the tail of the short axis LSP resonance in the TM spectrum.

a)

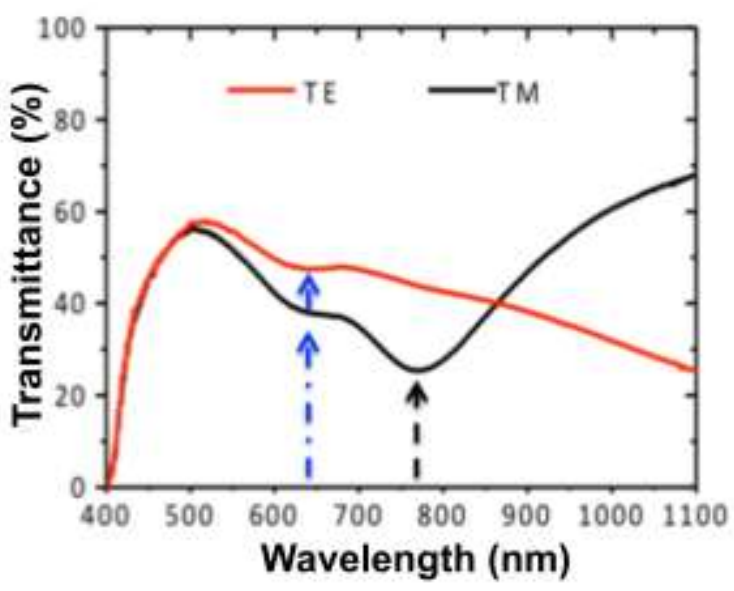

b)

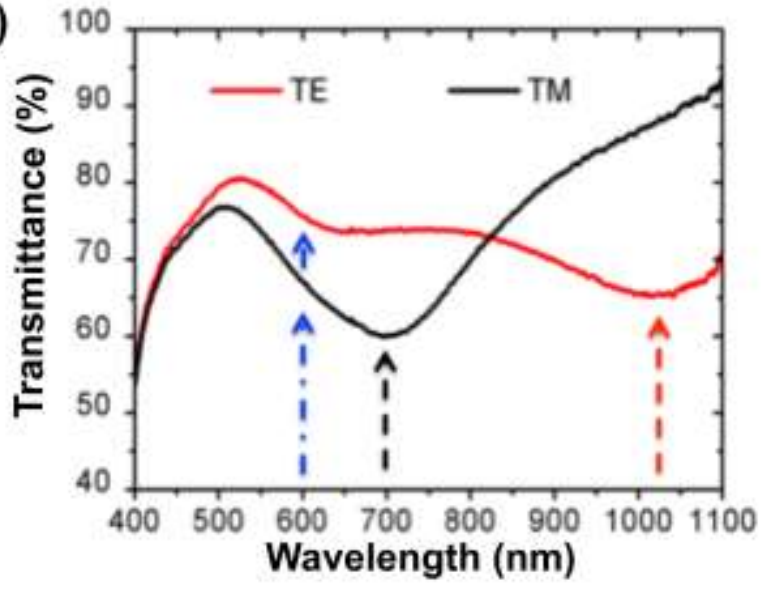

Figure S1 $(\boldsymbol{a}, \boldsymbol{b})$ Optical extinction spectra of Au nanocrescent arrays respectively confined on polystyrene monolayer at the incidence angle $\theta=70^{\circ}$ (spheres diameter $260 \mathrm{~nm}$ - graph adapted from Ref. [Error! Bookmark not defined.]) and $\theta=80^{\circ}$ (spheres diameter $300 \mathrm{~nm}$ ).

In Figure S2 we show the optical transmittance $(\mathrm{T})$ of Au nanocrescent arrays $(\mathrm{d}=10 \mathrm{~nm})$ for TE and TM polarization and the corresponding evaluation of the optical extinction $(\mathrm{E})$, due to plasmonic excitation. At the wavelength of $700 \mathrm{~nm}$ the plasmonic extinction respectively reads $\mathrm{E}_{\mathrm{TE}} \cong 2 \%$ and $\mathrm{E}_{\mathrm{TM}} \cong 21 \%$. From these values we evaluated the reflectance $(\mathrm{R}=1-\mathrm{T}-\mathrm{E})$ at the same wavelength thus obtaining $\mathrm{R}_{\mathrm{TE}} \cong 24 \%$ and $\mathrm{R}_{\mathrm{TM}} \cong 21 \%$. From this estimate one would conclude that due to the anisotropic reflectivity the dichroic SERS amplification should be of the order of $3 \%$ in favour of the TE-mode. We stress that this estimate though qualitative allows to conclude that the 
contribution of anisotropic reflectivity to SERS dichroism is small in absolute value and above all in the opposite direction with respect to the experimental observations.

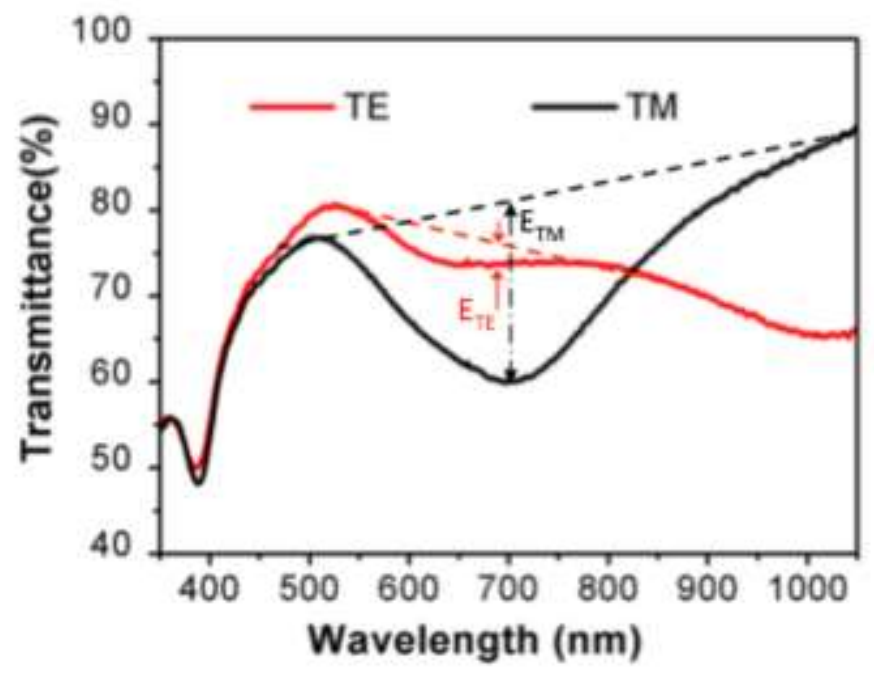

Figure S2 Measured optical transmission spectra of Au nanocrescent arrays (adapted from Figure 2(d) of the paper). The red and black double headed arrows represent the estimate of the optical extinction due to plasmonic excitation for TM and TE polarization $\left(E_{T M}, E_{T E}\right)$, at a wavelength of $700 \mathrm{~nm}$. 